\title{
CONSTITUINTES QUÍMICOS DE Galianthe brasiliensis (RUBIACEAE)
}

\author{
Vagner Marques de Moura, Daniela Pereira dos Santos e Silvana Maria de Oliveira Santin \\ Departamento de Química, Universidade Estadual de Maringá, Av. Colombo, 5790, 87020-900 Maringá - PR, Brasil \\ João Ernesto de Carvalho e Mary Ann Foglio \\ Centro Pluridisciplinar de Pesquisas Químicas, Biológicas e Agrícolas, CP 6171, 13083-970 Campinas - SP, Brasil
}

Recebido em 27/1/05; aceito em 28/9/05; publicado na web em 14/3/06

\begin{abstract}
CHEMICAL CONSTITUENTS OF Galianthe brasiliensis (Spreng.) E.L.Cabral \& Bacigalupo (RUBIACEAE). This paper describes the chemical constituents isolated from aerial parts of the plant Galianthe brasiliensis. From a methanol extract, the iridoid glycosides asperuloside, deacetylasperuloside, mixture of $Z$ - and $E$-6-O-p-coumaroylscandoside methyl ester, the triterpene ursolic acid and the steroids stigmasterol, campesterol, $\beta$-sitosterol and 3-O- $\beta$-glycopiranosyl sitosterol were isolated. The structures of the natural products were identified on the basis of spectral data, including 2D NMR experiments. The antiproliferative properties of the crude methanolic extract were investigated against a series of nine human cancer cell lines.
\end{abstract}

Keywords: Galianthe brasiliensis; chemical constituents; antiproliferative assays.

\section{INTRODUÇÃO}

Galianthe brasiliensis (Spreng.) E.L. Cabral \& Bacigalupo (sinonímias: Diodia brasiliensis Spreng. e D. polymorpha Cham. \& Schl.) é uma planta ornamental de pequeno porte, nativa do Brasil e conhecida pelo nome de poaia-do-campo ${ }^{1}$. Esta espécie é utilizada na medicina popular por suas propriedades eméticas ${ }^{2}$.

A partir de levantamento bibliográfico, constatamos a ausência de estudos químicos ou farmacológicos sobre esta espécie. Plantas do gênero Diodia são empregadas na medicina popular e tradicional como agentes anti-reumático, antidiarréico, antiofídico, laxativo e emético, e também no tratamento de úlceras gástricas, urticárias, dores de ouvido, dores abdominais e gastrites ${ }^{3,4}$. Estudos farmacológicos das espécies $D$. scandens ${ }^{3}$ e D. sarmentosa ${ }^{4}$ revelaram atividades antiinflamatória, antiúlcera, analgésica e antiofídica. Um único estudo químico encontrado para o gênero foi da espécie $D$. teres, o qual indicou a presença dos iridóides glicosilados asperulosídeo, ácido deacetilasperulosídeo e ácido geniposídico; da cumarina escopolina e dos flavonóides rutina, canferol-3-O-rutinosídeo, quercitrina, astragalina, isoquercitrina e quercetina ${ }^{5}$.

O presente trabalho descreve os resultados obtidos do estudo químico de G. brasiliensis, bem como a avaliação preliminar antiproliferativa do extrato bruto metanólico.

\section{RESULTADOS E DISCUSSÃO}

O fracionamento do extrato metanólico das partes aéreas de Galianthe brasiliensis resultou no isolamento de uma mistura dos esteróides sitosterol, estigmasterol e campesterol, do sitosterol, do 3-O- $\beta$-glicopiranosil sitosterol, do ácido ursólico ${ }^{6}$ e dos iridóides glicosilados asperulosídeo (1), deacetilasperulosídeo (2) e da mistura dos ésteres metílicos dos isômeros Z- e E-6-O-p-coumaroilescandosídeo (3-4) (Figura 1). As estruturas das substâncias isoladas foram elucidadas com base nas análises de dados de IV, EM, RMN ${ }^{1} \mathrm{He} \mathrm{e}{ }^{13} \mathrm{C}$ e de experimentos de DEPT $135^{\circ},{ }^{1} \mathrm{Hx}{ }^{1} \mathrm{H}$ COSY e HETCOR.

A substância 1 foi isolada das frações clorofórmio e clorofór-

*e-mail: smoliveira@uem.br

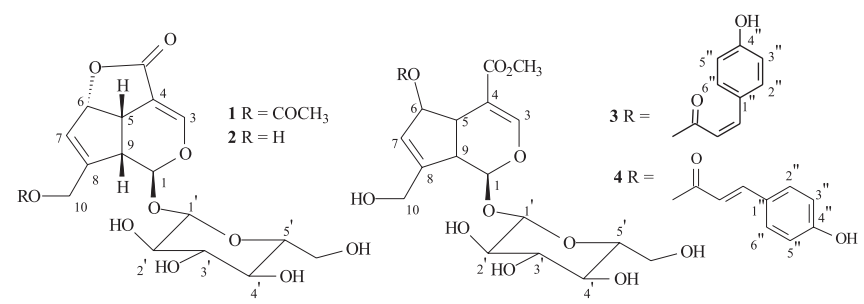

Figura 1

mio/metanol 1:1, como um sólido castanho amorfo. O espectro na região do infravermelho (IV) de $\mathbf{1}$ apresentou bandas de absorção típicas de grupos hidroxila $\left(3361 \mathrm{~cm}^{-1}\right)$ e carboxila de éster (1729 $\left.\mathrm{cm}^{-1}\right)$ além de uma lactona $\alpha, \beta$ insaturada $\left(1713\right.$ e $\left.1659 \mathrm{~cm}^{-1}\right)$. Os dados espectroscópicos de RMN desta substância, evidenciaram a presença de um esqueleto iridoidal caracterizado pelos sinais $\delta_{\mathrm{H}} / \delta_{\mathrm{C}} \mathrm{em} 7,30(d, J=2,4 \mathrm{~Hz}, \mathrm{H}-3) / 150,4(\mathrm{C}-3) ; 5,96(d, J=1,5 \mathrm{~Hz}$, $\mathrm{H}-1) / 93,4$ (C-1); 5,73 ( $s l, \mathrm{H}-7) / 129,1$ (C-7); 5,56 (dt, $J=6,6$ e 1,5 $\mathrm{Hz}, \mathrm{H}-6) / 86,4$ (C-6). O carbono carboxílico da lactona foi evidenciado pelo sinal em $\delta_{\mathrm{C}} 172,4$. A presença da unidade $-\mathrm{CH}_{2} \mathrm{OAc}$ ligada ao carbono insaturado (C-8) foi estabelecida pelos sinais observados em $\delta_{C} 61,9,172,8$ e 20,6 , respectivamente. Os sinais na região entre $\delta_{\mathrm{H}} 3,15-3,94$ em conjunto com o sinal em $\delta_{\mathrm{H}} 4,68(d$, $J=7,8 \mathrm{~Hz}, \mathrm{H}-1^{\prime}$ ) determinaram a presença de uma unidade D- $\beta$ glucose. Estas atribuições foram confirmadas após análises dos espectros bidimensionais (2D) de correlação homonuclear ${ }^{1} \mathrm{H}-{ }^{1} \mathrm{H}$ COSY e heteronuclear HETCOR, conforme Tabela 1. A estereoquímica relativa da substância foi determinada por experimentos de diferença de NOE. A irradiação do sinal correspondente a H-6 $\left(\delta_{\mathrm{H}} 5,56\right)$ produziu um aumento de intensidade dos sinais em $\delta_{\mathrm{H}} 3,65(\mathrm{H}-5)$ e $5,73(\mathrm{H}-7)$, e a irradiação do sinal correspondente a $\mathrm{H}-9\left(\delta_{\mathrm{H}} 3,28\right)$ produziu aumento de intensidade dos sinais correspondentes a H-5 e H-10a $\left(\delta_{\mathrm{H}} 4,77\right)$. A configuração em cis entre C5 e C-9 é comum para todos os iridóides que contêm o esqueleto básico carbocíclico ${ }^{7}$. A comparação dos dados espectroscópicos da substância isolada com os descritos na literatura ${ }^{8}$ permitiu a caracterização da estrutura como sendo o asperulosídeo (1).

A substância 2 foi isolada da fração clorofórmio/metanol 1:1 
Tabela 1. Dados RMN de ${ }^{1} \mathrm{H}(300 \mathrm{MHz})$ e correlações H-C (HETCOR) e H-H (COSY), para as substâncias 1 (CD 3 OD) e 2 (D 2 )

\begin{tabular}{|c|c|c|c|c|c|c|}
\hline & & 1 & & & 2 & \\
\hline & $\begin{array}{l}\text { HETCOR } \\
\left(\delta_{\mathrm{C}} / \mathrm{DEPT}\right) \\
\end{array}$ & $\delta_{\mathrm{H}}$ (mult.; $J \mathrm{~Hz}$ ) & (COSY H-H) & $\begin{array}{l}\text { HETCOR } \\
\left(\delta_{C} / \mathrm{DEPT}\right) \\
\end{array}$ & $\delta_{\mathrm{H}}($ mult.; $J \mathrm{~Hz})$ & (COSY H-H) \\
\hline 1 & $93,4 \mathrm{CH})$ & $5,96(d ; 1,5)$ & 3,28 (H9) & $95,9(\mathrm{CH})$ & $5,96(d ; 1,5)$ & \\
\hline 3 & $150,4(\mathrm{CH})$ & $7,30(d ; 2,4)$ & 3,65 (H5) & $153,4(\mathrm{CH})$ & $7,42(d ; 2,1)$ & \\
\hline 4 & $106,3(\mathrm{C})$ & & & $107,9(\mathrm{C})$ & & \\
\hline 5 & $37,4(\mathrm{CH})$ & $\begin{array}{l}3,65(t d ; 6,6 \\
\quad \text { e } 2,4)\end{array}$ & $\begin{array}{l}7,30(\mathrm{H} 3) ; 5,56 \\
\text { (H6); 3,28 (H9) }\end{array}$ & $38,8(\mathrm{CH})$ & $3,71(d t ; 6,9$ e 2,1$)$ & $\begin{array}{l}5,73(\mathrm{H} 6) \\
3,39 \text { (H9) }\end{array}$ \\
\hline 6 & $86,4(\mathrm{CH})$ & $\begin{array}{l}5,56(d t ; 6,6 \\
\quad \text { e } 1,5)\end{array}$ & $\begin{array}{c}3,65(\mathrm{H} 5) ; \\
3,28(\mathrm{H} 9) ; 4,65(\mathrm{H} 10 \mathrm{~b})\end{array}$ & $89,7(\mathrm{CH})$ & $5,73(s l)$ & $3,71(\mathrm{H} 5)$ \\
\hline 7 & $129,1(\mathrm{CH})$ & $5,73(s l)$ & 3,28 (H9); 4,65 (H10a) & $127,8(\mathrm{CH})$ & $5,71(s l)$ & 4,25 (H10) \\
\hline 8 & $144,5(\mathrm{C})$ & & & $150,4(\mathrm{C})$ & & \\
\hline 9 & $45,3(\mathrm{CH})$ & $3,28(m)$ & $\begin{array}{c}5,96(\mathrm{H} 1) ; 5,73 \\
(\mathrm{H} 7) ; 3,65(\mathrm{H} 9) ; \\
5,56(\mathrm{H} 6)\end{array}$ & $46,1(\mathrm{CH})$ & 3,39(m) & $\begin{array}{l}3,71(\mathrm{H} 5) \\
5,71(\mathrm{H} 7)\end{array}$ \\
\hline 10 & $61,9\left(\mathrm{CH}_{2}\right)$ & $\begin{array}{l}\text { (a) } 4,65(d q \text {; } \\
14,3 \text { e } 1,5) \\
\text { (b) } 4,77(d q \text {; } \\
14,3 \text { e } 1,5)\end{array}$ & $\begin{array}{c}5,73(\mathrm{H} 7) ; \\
5,56(\mathrm{H} 6), 4,77(\mathrm{H} 10 \mathrm{~b}) \\
5,73(\mathrm{H} 7) ; 5,56(\mathrm{H} 6) \\
4,77(\mathrm{H} 10 \mathrm{a})\end{array}$ & $61,6\left(\mathrm{CH}_{2}\right)$ & $4,25(s l)$ & $5,71(\mathrm{H} 7)$ \\
\hline 11 & $172,4(\mathrm{C})$ & & & $176,8(\mathrm{C})$ & & \\
\hline 12 & $172,8(\mathrm{C})$ & & & & & \\
\hline 13 & $20,6\left(\mathrm{CH}_{3}\right)$ & $2,07(s)$ & & & & \\
\hline 1 ' & $100,1(\mathrm{CH})$ & $4,68(d ; 7,8)$ & 3,19 (H2') & $101,6(\mathrm{CH})$ & $4,85(d ; 7,8)$ & 3,31 (H2') \\
\hline 2' & 74,7 (CH) & $3,19(d d ; 9,0$ e 7,8$)$ & 4,68 (H1'); 3,37 (H3’) & 75,6 (CH) & $3,31(d d ; 9,3$ e 8,1$)$ & $\begin{array}{l}\text { 4,85 (H1'); } \\
3,53\left(\mathrm{H} 33^{\prime}\right)\end{array}$ \\
\hline 3' & 77,9 (CH) & $3,37(t ; 9,3)$ & 3,19 (H2'), 3,25 (H4') & $78,5(\mathrm{CH})$ & $3,53(t ; 9,3)$ & 3,31 (H2’) \\
\hline $4^{\prime}$ & $71,6(\mathrm{CH})$ & $3,25(d d ; 9,3$ e 9,0$)$ & 3,37 (H3'); 3,35 (H-5') & $72,6(\mathrm{CH})$ & $3,41(d d ; 9,6$ e 9,0$)$ & 3,54 (H5') \\
\hline 5' & 78,4 (CH) & $\begin{array}{c}3,35(d d d ; 9,3 \\
6,3 \text { e } 2,1)\end{array}$ & $\begin{array}{l}\text { 3,92 (H6’a); 3,66 } \\
\text { (H6’b); 3,25 (H4') }\end{array}$ & $79,4(\mathrm{CH})$ & $3,54(d d ; 9,6$ e 2,1$)$ & 3,41 (H4') \\
\hline $6^{\prime}$ & $62,8\left(\mathrm{CH}_{2}\right)$ & $\begin{array}{l}\text { (a) } 3,92(d d \\
11,7 \text { e } 2,1)\end{array}$ & $\begin{array}{c}\text { 3,35 (H5'); 3,66 } \\
\left(\mathrm{H} 6{ }^{\prime} \mathrm{b}\right)\end{array}$ & $63,7\left(\mathrm{CH}_{2}\right)$ & $3,96(d d ; 12,1$ e 2,1$)$ & $\begin{array}{l}\text { 3,54 (H5'); } \\
\text { 3,75 (H6'b) }\end{array}$ \\
\hline & & $\begin{array}{l}\text { (b) } 3,66(d d \text {; } \\
11,7 \text { e } 6,3)\end{array}$ & 3,35 (H5'); 3,92 (H6'a) & & $3,75(d d, 12,1$ e 6,0$)$ & 3,96 (H6’a) \\
\hline
\end{tabular}

como um sólido castanho amorfo. O espectro no IV de 2 apresentou bandas de absorção dos grupos hidroxila $\left(3380 \mathrm{~cm}^{-1}\right)$ e carboxila de éster $\left(1738 \mathrm{~cm}^{-1}\right)$. Os espectros de $\mathrm{RMN}{ }^{1} \mathrm{H}$ e ${ }^{13} \mathrm{C}$ mostraram sinais com deslocamentos químicos semelhantes àqueles apresentados para o asperulosídeo (1), diferenciando apenas na ausência de um grupo acetila em $\mathrm{C}$-10 (neste substituído pelo grupo $\mathrm{OH}$ ), que pode ser evidenciado pela diferença de deslocamento de H-10 e ainda, pela presença de apenas um sinal em $\delta_{C} 176,8$. Estas informações aliadas com a análise dos dados de COSY e HETCOR permitiram atribuir os deslocamentos de todos os hidrogênios e carbonos desta substância (Tabela 1). A comparação destes dados com os dados de $\mathbf{1}$ (como modelo) e os da literatura ${ }^{9}$ permitiram identificar a substância isolada como deacetilasperulosídeo.

$\mathrm{O}$ espectro de $\mathrm{RMN}$ de ${ }^{1} \mathrm{H}$ de $\mathbf{3}$ e $\mathbf{4}$ apresentou sinais em duplicidade e com intensidades relativas diferentes, indicando a presença de duas substâncias. A presença de D- $\beta$-glucose foi observada para ambas as substâncias através de dois dubletos em $\delta 4,71(\mathrm{~J}=$ $7,8 \mathrm{~Hz})$ e $4,79(J=7,8 \mathrm{~Hz})$. Foram ainda observados dois grupos de sinais em $\delta 6,36(d, J=16 \mathrm{~Hz})$ e $7,65(d, J=16 \mathrm{~Hz})$, e $\delta 5,81(d, J$ $=12,6 \mathrm{~Hz})$ e $6,87(d, J=12,6 \mathrm{~Hz})$ que indicaram a existência de ligações duplas trans e cis respectivamente. Os sinais de hidrogênios aromáticos foram observados como dubletos em $\delta 6,80(J=$ $8,7 \mathrm{~Hz})$ e $7,47(J=8,7 \mathrm{~Hz})$. Estes foram acompanhados por sinais similares menores em $\delta 6,72(J=9,0 \mathrm{~Hz})$ e 7,60 $(J=9,0 \mathrm{~Hz})$, relativos ao isômero cis. A razão trans:cis foi calculada em aproxi- madamente 70:30. A presença de núcleo iridoidal foi evidenciada pelos sinais em $\delta 7,28(d, J=1,2 \mathrm{~Hz}), 5,0(d, J=9,0 \mathrm{~Hz}), 5,81 \mathrm{e}$ 5,99 como singletos largos para a substância majoritária (4), e pelos sinais em $\delta 7,26(d, J=1,2 \mathrm{~Hz}), 4,92(d, J=5,4 \mathrm{~Hz}), 5,81$ e 5,97 como singletos para a substância em quantidade minoritária (3), correspondentes a H-3, H-1, H-6 e H-7, respectivamente. A presença dos sinais em $\delta 3,29$ e 3,30 como singletos (para 3 e 4) juntamente com os valores de deslocamentos observados para $\mathrm{H}-3$ indicaram a presença de grupo carboximetila para C-11. Os pares de sinais de $\mathrm{RMN}{ }^{13} \mathrm{C}$ e em $\delta 127,4 ; 133,7 ; 116,9$ e 161,4 e em $\delta$ 127,$4 ; 131,3 ; 115,4$ e 161,4 (correspondentes a C-1", C-2"e 6", C3 " e 5", e C-4" para 3 e 4) confirmaram a presença de grupos coumaroila. Os deslocamentos químicos de H-6 $(\delta 5,81)$ e H-10 (3: $\delta 4,17$ e 3,$83 ; 4: 4,38$ e 3,87 ) sugerem os grupos coumaroilatos ligados a C-6 de cada derivado 3 e 4 . A análise destes dados e comparações com dados da literatura ${ }^{10}$ permitiram as caracterizações das estruturas como sendo dos ésteres metílicos de $Z$ - e $E$-6$O$-p-coumaroilescandosídeo. Estas substâncias, juntamente com asperulosídeo, estão presentes em Hedyotis diffusa (Rubiaceae), espécie usada na medicina chinesa no combate a vários tumores ${ }^{10}$. A presença destes iridóides em Galianthe brasiliensis está de acordo com o posicionamento botânico e as correlações quimiossistemáticas do gênero na subfamília Rubioideae ${ }^{11}$.

O extrato bruto das partes aéreas de G. brasiliensis foi submetido à avaliação da atividade antiproliferativa em culturas de células 
tumorais humanas de melanoma (UACC62), mama (MCF7), pulmão (NCI460), leucemia (K562), ovário (OVCAR), próstata (PCO3), cólon (HT29), rim (786-0) e mama resistente (NCIADR), esta última expressa o fenótipo de resistência a múltiplos fármacos. $\mathrm{O}$ extrato apresentou efeito citostático (inibição de crescimento) e efeito citocida (morte celular) com boa seletividade entre as linhagens estudadas. Foi observado efeito citostático na maior concentração testada $(250 \mu \mathrm{g} / \mathrm{mL})$ para as linhagens de mama, ovário e mama resistente, com inibições de crescimento em 100, 70 e 53\%, respectivamente. Para a linhagem K562 (leucemia linfóide), o extrato apresentou inibição de crescimento de $66 \%$ na concentração de $25 \mu \mathrm{g} / \mathrm{mL}$ e ainda, morte celular de $65 \%$ na maior concentração testada. Como controle positivo das linhagens testadas foi utilizado o quimioterápico doxorrubicina. Na comparação com os resultados obtidos para as duas avaliações, constatou-se efeito similar citocida e citostático entre as mesmas, com melhor seletividade para o extrato. A efetiva atividade antiproliferativa observada para o extrato bruto das partes aéreas de Galianthe brasiliensis pode ser relacionada, em parte, às substâncias ácido ursólico ${ }^{12}$ e asperulosídeo ${ }^{13}$ presentes, que possuem comprovada atividade anti-tumoral.

\section{PARTE EXPERIMENTAL}

\section{Procedimentos experimentais gerais}

Os espectros de absorção na região do infravermelho (IV) foram registrados em um espectrofotômetro Bomem, modelo MBséries, em pastilha de $\mathrm{KBr}$, na região de 400 a $4000 \mathrm{~cm}^{-1}$. Utilizouse absorção em $1028 \mathrm{~cm}^{-1}$ de um filme de poliestireno como referência. Os espectros de massas de baixa resolução foram obtidos em espectrômetro Shimadzu-CG/MS modelo QP 2000A operando pelo modo de ionização por impacto eletrônico $(70 \mathrm{eV})$, equipado com probe para sólidos. Os espectros de $\mathrm{RMN}$ de ${ }^{1} \mathrm{H}$ e ${ }^{13} \mathrm{C}$ foram obtidos em espectrômetro Varian modelo Gemini 2000, operando a $300 \mathrm{MHz}$ para ${ }^{1} \mathrm{H}$ e $75,5 \mathrm{MHz}$ para ${ }^{13} \mathrm{C}$, tendo como referência interna o tetrametilsilano (TMS) ou o próprio solvente. Os deslocamentos químicos $(\delta)$ foram registrados em ppm e os solventes deuterados utilizados foram $\mathrm{CDCl}_{3}, \mathrm{CD}_{3} \mathrm{OD}, \mathrm{C}_{5} \mathrm{D}_{5} \mathrm{~N}$ e $\mathrm{D}_{2} \mathrm{O}$. Nas cromatografias em coluna (CC) utilizaram-se colunas de vidro de dimensões variadas e como fase estacionária, sílica gel 60 (0,063 0,200 mm) da Merck e sílica gel 60 (0,04 - 0,063 mm) da Fluka.

Nas cromatografias em camada delgada analítica (CCDA) foram utilizadas placas de vidro contendo sílica gel 60 GF254 da Merck e para a visualização dos compostos nas mesmas, utilizouse radiação no ultravioleta $\lambda=254$ e $366 \mathrm{~nm}$, seguida de nebulização com solução de $\mathrm{H}_{2} \mathrm{SO}_{4} / \mathrm{MeOH}$ (1:1) ou $\mathrm{H}_{2} \mathrm{SO}_{4}$ /anisaldeído/ácido acético (1:0,5:50) e levadas a aquecimento.

\section{Material vegetal}

A espécie vegetal Galianthe brasiliensis foi coletada às margens da bacia de inundação do rio Paraná na região de Porto Rico/ PR, em dezembro de 1997. A excicata do material vegetal encontra-se depositada no Herbário da UEM, sob registro HUM 3401.

\section{Extração e isolamento dos constituintes químicos}

As partes aéreas secas ao ar e moídas $(315,00 \mathrm{~g})$ foram submetidas à percolação em metanol, à temperatura ambiente. A evaporação do solvente sob vácuo em evaporador rotativo resultou no extrato bruto metanólico $(44,15$ g). Parte deste extrato $(20,10$ g) foi submetida a uma semipurificação em coluna cromatográfica de sílica gel utilizando como eluentes hexano $(\mathbf{H} ; 0,21 \mathrm{~g})$, hexano/ clorofórmio 1:1 (HC; 0,85 g), clorofórmio (C; 1,63 g), clorofórmio/etanol 1:1 (CE; 5,83 g) e etanol (E; 6,67 g).

A fração $\mathbf{H C}(0,85 \mathrm{~g})$ foi submetida à cromatografia em coluna (CC) de sílica gel, utilizando-se como eluentes hexano e misturas de hexano/AcOEt em gradiente de polaridade. A purificação da fração hexano/AcOEt 95;0,5 (224,2 mg) por CC em sílica gel e hexano como eluente resultou no isolamento do esteróide sitosterol (98,7 mg). O tratamento da fração hexano/AcOEt 9:1 (100,0 mg) por CC de sílica gel (hexano, hexano/AcOEt 2-20\%; 6 frações), seguida da recristalização em acetona da subfração 4 resultou na obtenção das substâncias sitosterol, estigmasterol e campesterol como uma mistura (10,0 mg). A fração AcOEt (111,4 mg), resultante do tratamento de $\mathbf{H C}$, foi cromatografada em CC de sílica gel $\left(\mathrm{CHCl}_{3}, \mathrm{CHCl}_{3} / \mathrm{MeOH} 3-10 \%\right)$, obtendo-se a substância 3-O$\beta$-glicopiranosil sitosterol (11,4 mg).

A fração C (1,63 g) foi levada a uma separação em CC de sílica gel eluída com hexano/AcOEt em gradiente crescente de polaridade obtendo-se 140 frações de $30 \mathrm{~mL}$ cada, reunidas em 9 subfrações. A subfração C-4 (339,8 mg) foi recromatografada em coluna de sílica gel (AcOEt e AcOEt/MeOH 5-20\%; 7 frações), e a subfração C-4.3 forneceu o triterpeno ácido ursólico $(15,9 \mathrm{mg})$.

A fração CE (5,70 g) foi fracionada em coluna de sílica gel, eluída com AcOEt/MeOH em gradiente crescente de polaridade, obtendo-se 150 frações de $30 \mathrm{~mL}$ cada e reunidas em 8 subfrações. Da subfração CE-4, após sucessivas cromatografias em coluna de sílica gel utilizando-se como eluente $\mathrm{AcOEt} / \mathrm{MeOH} 5 \%$, isolou-se a substância 1 (41,8 mg). A CC de sílica gel da subfração CE-6 (453,3 $\mathrm{mg}$ ) utilizando como eluentes $\mathrm{CHCl}_{3} / \mathrm{MeOH}$ em gradiente crescente de polaridade (5-20\%) resultou em 65 frações de $30 \mathrm{~mL}$ cada e reunidas em 6 subfrações. A subfração CE-6.1 foi submetida a uma CC de sílica gel $\left(\mathrm{CHCl}_{3} / \mathrm{MeOH} 5-10 \%, 4\right.$ subfrações) sendo que a subfração CE-6.1.2 forneceu novamente o ácido ursólico (18,7 mg). A subfração CE-6.5 (209,7 mg) foi sucessivamente cromatografada em coluna de sílica gel $\left(\mathrm{CHCl}_{3} / \mathrm{MeOH}\right.$ 9,5:0,5; 9:1 e 8,5:1,5) fornecendo a substância 2 (12,5 mg) e também após sucessivas lavagens com acetona a frio dos cristais presentes na subfração CE-6.5.5 (15,1 $\mathrm{mg}$ ) obteve-se os compostos isômeros 3-4 (4,0 mg).

Asperulosídeo (1): sólido castanho amorfo. IV $v_{\text {máx }}(\mathrm{KBr}) \mathrm{cm}^{-1}$ : 3361, 1729 e 1713, 1632. RMN ${ }^{1} \mathrm{H}$ e ${ }^{13} \mathrm{C}$ : Tabela 1.

Deacetilasperulosídeo (2): sólido castanho amorfo. IV $v_{\text {máx }}$. $(\mathrm{KBr}) \mathrm{cm}^{-1}$ : 3380, 1738, 1659. RMN ${ }^{1} \mathrm{H}^{13} \mathrm{C}$ : Tabela 1.

Éster metílico de Z-6-O-p-coumaroilescandosídeo (3): RMN ${ }^{1} \mathrm{H}\left(300 \mathrm{MHz}, \mathrm{CD}_{3} \mathrm{OD}\right) \delta \mathrm{ppm}$ (multiplicidade, $\left.J\right): 4,92(d, J=5,4$ $\mathrm{Hz}, 1 \mathrm{H}, \mathrm{H}-1), 7,26$ (d, $J=1,2 \mathrm{~Hz}, 1 \mathrm{H}, \mathrm{H}-3), 3,23$ (d, $J=6,6$ 1H, H5), 5,81 ( $s l, 1 \mathrm{H}, \mathrm{H}-6), 5,97$ ( $s l, 1 \mathrm{H}, \mathrm{H}-7), 2,84$ (dd, $J=6,3$ e $6,6 \mathrm{~Hz}$, $1 \mathrm{H}, \mathrm{H}-9), 4,17$ (d, $J=15,9 \mathrm{~Hz}, 1 \mathrm{H}, \mathrm{H}-10), 3,83(d, J=15,9 \mathrm{~Hz}, 1 \mathrm{H}$, H-10) 3,29 (s, 3H, H-12), 4,71 (d, $J=7,8 \mathrm{~Hz}, 1 \mathrm{H}, \mathrm{H}-1$ ') $, 3,03$ (m, 1H, H-2'), 3,31 ( $m, 1 \mathrm{H}, \mathrm{H}-3$ '), 3,20 ( $m, 1 \mathrm{H}, \mathrm{H}-4$ ') , 3,25 ( $m, 1 \mathrm{H}, \mathrm{H}-$ 5'), 3,79 ( $d, J=12,3 \mathrm{~Hz}, 1 \mathrm{H}, \mathrm{H}-6$ '), 6,87 ( $d, J=12,6 \mathrm{~Hz}, 1 \mathrm{H}, \mathrm{H}-\alpha)$, $5,81(d, 12,6 \mathrm{~Hz}, 1 \mathrm{H}, \mathrm{H}-\beta), 7,60(d, J=9,0 \mathrm{~Hz}, 2 \mathrm{H}, \mathrm{H}-2$ "'/H-6"), 6,72 ( $d, J=9.0,2 \mathrm{H}, \mathrm{H}-3$ "'/H-5"); RMN ${ }^{13} \mathrm{C}$ (75.5 MHz, CD $\left.\mathrm{OD}\right) \delta$ ppm: 98,7 (C-1), 150,1 (C-3), 108,9 (C-4), 43,2 (C-5), 83,2 (C-6), 129,7 (C-7), 147,0 (C-8), 46,6 (C-9), 61,6 (C-10), 168,7 (C-11), 56,0 (C-12), 99,6 (C-1'), 74,8 (C-2'), 77,9 (C-3'), 70,6 (C-4'), 78,5 (C-5'), 62,5 (C-6'), 146,5 (C- $\alpha), 116,9$ (C- $\beta$ ), 127,4 (C-1”), 133,7 (C-2"/C-6"), 116,9 (C-3"/C-5"), 161,4 (C-4"), 167,7 (CO).

Éster metílico de E-6-O-p-coumaroilescandosídeo (4): $\mathrm{RMN}{ }^{1} \mathrm{H}$ (300 $\left.\mathrm{MHz}, \mathrm{CD}_{3} \mathrm{OD}\right) \delta \mathrm{ppm}$ (multiplicidade, $\left.J\right): 5,00(d, J=9,0 \mathrm{~Hz}$, $1 \mathrm{H}, \mathrm{H}-1), 7,28$ ( $d, J=1,2 \mathrm{~Hz}, 1 \mathrm{H}, \mathrm{H}-3), 3,23$ (d, $J=6,9 \mathrm{~Hz}, 1 \mathrm{H}, \mathrm{H}-5)$, $5,81$ ( $s l, 1 \mathrm{H}, \mathrm{H}-6), 5,99(s l, 1 \mathrm{H}, \mathrm{H}-7), 2,87(d d, J=6,3$ e $6,6 \mathrm{~Hz}, 1 \mathrm{H}$, H-9), 4,38 ( $d, J=15,9 \mathrm{~Hz}, 1 \mathrm{H}, \mathrm{H}-10), 3,87$ ( $d, J=15,9 \mathrm{~Hz}, 1 \mathrm{H}, \mathrm{H}-$ 10), 3,30 ( $s, 3 \mathrm{H}, \mathrm{H}-12), 4,79$ ( $d, J=7,8 \mathrm{~Hz}, 1 \mathrm{H}, \mathrm{H}-1$ ') $, 3,03$ ( $m, 1 \mathrm{H}$, H-2'), 3,31 ( $\left.m, 1 \mathrm{H}, \mathrm{H}-3^{\prime}\right), 3,20$ ( $\left.m, 1 \mathrm{H}, \mathrm{H}-4^{\prime}\right), 3,25$ ( $m, 1 \mathrm{H}, \mathrm{H}-5$ '), 
$3,79(d, J=12,3 \mathrm{~Hz}, 1 \mathrm{H}, \mathrm{H}-6$ ') $7,65(d, J=16,0 \mathrm{~Hz}, 1 \mathrm{H}, \mathrm{H}-\alpha), 6,36$ $(d, J=16,0 \mathrm{~Hz}, 1 \mathrm{H}, \mathrm{H}-\mathrm{b}), 7,47$ ( $d, J=8,7 \mathrm{~Hz}, 2 \mathrm{H}, \mathrm{H}-2 " / \mathrm{H}-6 "), 6,80$ (d, $J=8,7 \mathrm{~Hz}, 2 \mathrm{H}, \mathrm{H}-3$ "'/H-5"); RMN ${ }^{13} \mathrm{C}\left(75.5 \mathrm{MHz}, \mathrm{CD}_{3} \mathrm{OD}\right) \delta$ ppm: 98,9 (C-1), 150,1 (C-3), 108,9 (C-4), 43,2 (C-5), 83,2 (C-6), 129,7 (C-7), 147,6 (C-8), 46,6 (C-9), 61,6 (C-10), 168,7 (C-11), 56,0 (C-12), 99,9 (C-1'), 75,0 (C-2'), 77,9 (C-3'), 71,6 (C-4'), 78,5 (C5'), 62,5 (C-6'), 145,0 (C- $\alpha$ ), 115,8 (C- $\beta$ ), 127,4 (C-1"), 131,3 (C2"/C-6"), 115,4 (C-3"/C-5"), 161,4 (C-4"), 167,7 (CO)

\section{Avaliação da atividade antiproliferativa}

O extrato metanólico foi avaliado frente a culturas de células tumorais humanas de melanoma (UACC62), mama (MCF7), pulmão (NCI460), leucemia (K562), ovário (OVCAR), próstata (PCO3), cólon (HT29), rim (786) e mama resistente (NCIADR), segundo metodologia descrita por Monks e colaboradores ${ }^{14}$, utilizando doxorrubicina como controle positivo. $\mathrm{O}$ extrato foi avaliado nas concentrações de 0,$25 ; 2,5,25$ e $250 \mu \mathrm{g} / \mathrm{mL}$.

\section{AGRADECIMENTOS}

À Prof ${ }^{\mathrm{a}}$. Dra ${ }^{\mathrm{a}}$. M. C. de Souza (Departamento de Biologia, UEM) pela coleta e identificação da planta, à CAPES e ao CNPq pelas bolsas concedidas (V. M. de Moura e D. P. dos Santos).

\section{REFERÊNCIAS}

1. Lorenzi, H.; Souza, H. M.; Plantas Ornamentais no Brasil, Instituto Plantarum de Estudo da Flora Ltda: Nova Odessa, 1999, p. 955
2. Corrêa, M. P.; Dicionário das Plantas Úteis do Brasil e das Exóticas Cultivadas, Imprensa Nacional: Rio de Janeiro, 1984, vol. 5, p. 565.

3. Akah, P. A.; Okogun, J. I.; Ekpendu, T. O.; Phytother. Res. 1993, 7, 317; Akubue, P. I.; Langason, R. B. F.; Akunyili, D. N.; Fitoterapia 1994, 65, 235; Onuaguluchi, G.; Nwafor, P.; Phytother. Res. 1999, 13, 459.

4. Akah, P. A.; Orisakwe, O. E.; Gamaniel, K. S.; Shittu, A.; J. Ethnopharmacol. 1998, 62, 123.

5. Lee, J. H.; Ku, C. H.; Baek, N. I.; Kim, S. H.; Park, H. W.; Kim, D. K.; Arch. Pharm. Res. 2004, 27, 40.

6. Agrawal, P. K.; Phytochemistry 1992, 31, 3307; Matida, A. K.; Rossi, M. H.; Blumenthal, E. E. A.; Schuquel, I. T. A.; Malheiros, A.; Vidotti, G. J.; Anais Assoc. Bras. Quím. 1996, 45, 147; Mahato, S. B.; Kundu, A. P.; Phytochemistry 1994, 37, 1517.

7. Sampaio-Santos, M. I.; Kaplan, M. A. C.; J. Braz. Chem. Soc. 2001, 12, 144.

8. Breitmaier, E.; Structure Elucidation by NMR in Organic Chemistry, John Wiley \& Sons Ltda: Chichester, 1993, p. 217.

9. Lopes, S.; Poser, G.L.; Kerber, F. M. F.; Konrath, E. L.; Moreno, M. E. S.; Zuanazzi, J. A. S.; Henriques, A. T.; Biochem. Syst. Ecol. 2004, 32, 1187.

10. Nishihama, Y.; Masuda, K.; Yamaki, M.; Takagi, S.; Sakina, K.; Planta Med. 1981, 43, 28; Wu, H.; Tao, X.; Chen, Q.; Lao, X.; J. Nat. Prod. 1991, $54,254$.

11. Moura, V. M.; Santos, A. R.; Nurnberg, V.; Souza, M. C.; Santin, S. M. O.; Biochem. Syst. Ecol. 2005, 33, 451; Inouye, H.;Takeda, Y.; Nishimura, H.; Kanomi, A.; Okuda, T.; Puff, C.; Phytochemistry 1988, 27, 2591.

12. Hsu, H. Y.; Yang, J. J.; Lin, C.C.; Cancer Lett. 1997, 111, 7; Hsu, H. Y.; Kuo, P. L.; Lin, C.C.; Life Sci. 2004, 75, 2303.

13. Nakamura, T.; Nakazawa, Y.; Onizuka, S.; Satoh, S.; Chiba, A.; Sekihashi, K.; Miura, A.; Yasugahira, N.; Sasaki, Y.F.; Mutat. Res. 1997, 388, 7.

14. Monks, A.; Scudiero, D.; Skehan, P.; Shoemaker, R.; Paull, K.; Vistica, D.; Hose, C.; Langley, J.; Cronise, P.; Vaigrowolff, A.; Graygoodrich, M.; Campbell, H.; Mayo, J.; Boyd, M.; J. Nat. Cancer Inst. 1991, 83, 757. 